\title{
Análisis de las situaciones de juego 2vs2 en el campeonato europeo masculino de balonmano 2012: Aplicación de la técnica de coordenadas polares
}

\section{Analysis of 2vs2 situations in men's european handball championship 2012: Application of polar coordinates technique}

\section{Análise das situaçóes de jogo 2vs2 no campeonato europeu masculino de andebol 2012: Aplicação da técnica de coordenadas polares}

\author{
Duarte Jesus Sousa ${ }^{1 *}$, João Nunes Prudente ${ }^{1}$, Pedro Sequeira ${ }^{2}$, José Antonio López-López ${ }^{3}$ y Antonio Hernández-Mendo \\ ${ }^{1}$ Universidad de Madeira. Centro de Ciencias Sociales. Departamento de Educación Física y Deporte. Portugal, ${ }^{2}$ Unidad de Investigación del Instituto \\ Politécnico de Santarém-Escuela Superior de Deporte de Rio Maior. Portugal, ${ }^{3}$ Universidad de Málaga. España
}

\begin{abstract}
Resumen: Se analizan los patrones de juego en ataque en situaciones 2vs2, en igualdad 6vs6, ocurridos en los partidos de la fase final del Campeonato Europeo Masculino de Balonmano 2012. Se utilizan los partidos televisados para analizar los patrones de juego en ataque en situaciones 2 vs2 que se produjeron durante los partidos observados. Para ello, se utiliza como instrumento un formato de campo combinado con sistema de categorías, se emplea 16 partidos con un total de 1208 ataques y 390 situaciones de juego 2vs2. Se utiliza la técnica de análisis de coordenadas polares, utilizando el software Hoisan, el cual permite analizar las interrelaciones entre las distintas categorías que componen el sistema de observación. Los resultados muestran que existe probabilidad significativa de: 1) "Fijar" inhibe la "Falta de Atacante"; "Fijar/Desmarcar" activa la "Falta de Atacante" e inhibe "Falta Técnica" y la "Continuidad del Juego", pero es activada por la "Continuidad del Juego"; 2) "Bloqueo" activa "Gol" y "Falta de Atacante" inhibe "Lanzamiento 7 metros"; "Bloqueo con Continuidad" inhibe "Lanzamiento 7 metros" y "Parada de Portero" pero es activado por la "Parada de Portero"; 3) "Cruce con Finalización" activa "Lanzamiento 7 metros" y "Continuidad del Juego," inhibe la "Falta de Atacante". Activa también "Parada de Portero" y está inhibida por la misma; 4) "Cruce con Continuidad" inhibe "Lanzamiento Fuera" pero activa "Lanzamiento contra el Poste/Travesańo" siendo inhibidas por este. Los resultados muestran que el uso de 2vs2 puede influir en la eficacia del ataque, lo que es un indicativo claro para los entrenadores por la necesidad de practicar estas situaciones. También refuerzan la idea de la importancia del Pivote en estas situaciones de 2vs2, debiendo tenerse en cuenta en el entrenamiento.
\end{abstract}

Palabras clave: Análisis del rendimiento, Metodología Observacional, Análisis de Coordenadas Polares, Balonmano masculino de élite, comportamiento táctico, $2 \mathrm{vs} 2$.

Abstract: Broadcast footage of Men's European Handball Championship 2012 were used to analyze attacking patterns of play in the $2 \mathrm{vs} 2$ game situations that occurred during matches from final phase. An ad hoc instrument, field format combined with categories system, was used to gather data from 16 matches, which yielded 1208 attacks and 390 2vs2 game situations. Polar coordinates analysis was carried out by means of Hoisan software that enables us to analyze the interrelations between the different categories of

Dirección para correspondencia [Correspodence address]: Duarte Filipe Jesus Sousa. Centro de Ciências Sociais. Departamento de Educación Física y Deporte de la Universidad de Madeira. Campus Universitario de Penteada -9020-105 Funchal (Portugal). E-mail: duartesousaa@gmail.com the observation tool. The results show that is significant the probability of: 1) "Fix" inhibits "Attack Fault"; "Fix/Uncheck" activates "Attack Fault" and inhibits "Technical Fault" and "Continuity of Play" but is activated by the "Continuity of Play"; 2) "Lock" activate "Goal" and "Attack Fault" and inhibits "7 meters"; "Lock with Continuity" inhibits "7 meters" and "Goalkeeper Save" but is activated by him; 3) "Cross with Shot" activate "7 meters" and "Continuity of Play", inhibits "Attack Fault". Also activate "Goalkeeper Save" and is inhibited by this action; 4) "Cross with Continuity" inhibits "Shot out" but active "Shot against the Post" and is inhibited by him. The results show that the use of $2 \mathrm{vs} 2$ can influence the effectiveness of the attack, giving a clear indication to the coaches for the need to train these situations. Also reinforce the idea of the importance of the Pivot in these situations of $2 \mathrm{vs} 2$, which must be taken into account in training. Key words: Performance Analysis, Observational methodology, Polar coordinates, elite male handball, attack tactical behaviour, 2 vs 2 .

Resumo: Analisou-se os padrōes de jogo no ataque ocorridos nas situaçôes de 2vs2, em igualdade 6vs6, durante os jogos da fase final do Campeonato Europeu Masculino de Andebol 2012, recolhidos a partir de jogos televisionados. Um instrumento ad hoc, formato de campo com sistemas de categorias, foi utilizado para recolher os dados de 16 jogos, num total de 1208 ataques e 390 situaçóes de jogo 2vs2. Recorreu-se à técnica de análise de coordenadas polares, utilizando o software Hoisan, que nos permitiu analisar as interrelações entre as diferentes categorias componentes do instrumento de observação. Os resultados mostram que é significativa a probabilidade: 1) "Fixar" inibir a "Falta Atacante"; "Fixar/Desmarcar" ativar a "Falta Atacante" e inibir a "Falta Técnica" e "Continuidade de Jogo" mas ser ativada pela "Continuidade do Jogo"; 2) «Bloqueio" ativar "Golo" e "Falta Atacante" inibindo "7 metros"; "Bloqueio com Continuidade" inibir "7 metros" e "Defesa do Guarda-redes" mas ser ativado pela "Defesa do Guarda-redes"; 3) "Cruzamento com Finalização" ativar "7 metros" e "Continuidade do jogo" e inibir "Falta Atacante". Ativa também a "Defesa do Guarda-redes" sendo inibido por esta; 4) "Cruzamento com Continuidade» inibe "Remate para Fora" mas ativa "Remate contra Poste/Trave" sendo inibido por este. Os resultados revelam que a utilizaçấo do $2 \mathrm{vs} 2$ pode influenciar a eficácia do ataque, dando uma indicação clara aos treinadores para a necessidade de treinar estas situaçôes.. Reforçam também a ideia da importância do Pivot nestas situaçóes de 2vs2, o que deve ser tido em conta no treino.

Palavras-chave: Análise da Performance, Metodología Observacional, Análise de Coordenadas Polares, Andebol masculino de elite, comportamento tático, $2 \mathrm{vs} 2$. 


\section{Introducción}

Actualmente los entrenadores buscan entender qué factores permiten aumentar las posibilidades de ganar. A pesar de que cada vez más autores asocian la toma de decisiones, los médios tácticos de dos y tres jugadores y el comportamiento táctico-técnico al rendimiento y al éxito deportivo en el balonmano (Prudente, 2006; Ribeiro y Silva, 2002; Santos, 2004; Santos, 2012; Sequeira, 2012; Silva, 2008), la bibliografía sobre este tema es escasa.

Por lo tanto, la complejidad del nivel de juego, la continua dificultad de evaluar el rendimiento de los equipos y atletas y la búsqueda permanente de respuestas que pueden contribuir a un mejor rendimiento lleva a querer estudiar esta modalidad. Asimismo, se limita este estudio a la relación de cooperación entre dos jugadores existentes en el proceso ofensivo, ya que, como considera Antón García (2000), el 2vs2 es una forma básica de cooperación. En cuanto a esta temática, Prudente (2006) considera que durante el juego existen pequeńos duelos 1vs1, 2vs2 y 3vs3, coincidiendo con Róman-Seco (2005) y Sevim y Bilge (2005) en señalar la utilización de las situaciones de $1 \mathrm{vs} 1,2 \mathrm{vs} 2$ y $3 \mathrm{vs} 3$ como un medio para crear oportunidades para la finalización de las acciones ofensivas en ataque organizado.

El aumento de los estudios realizados en balonmano demuestra una búsqueda continua e incesante sobre los problemas planteados en el juego, las preguntas de los entrenadores sobre cómo preparar los equipos para responder mejor a estos problemas y la búsqueda de respuestas para encontrar una relación con el éxito deportivo. Por lo tanto, interesa estudiar el $2 \mathrm{vs} 2$, ya que apunta como uno de los factores asociados con el éxito de los equipos de Balonmano, y es este factor el que permite dar respuestas sobre esta temática (Antón García, 2000; Prudente, 2006; Román-Seco; 2005; Sevim y Bilge, 2005).

Siendo el balonmano una modalidad integrada en los Juegos Deportivos Colectivos, presenta características propias, como la complejidad y comportamientos interactivos donde el comportamiento táctico-técnico se asume como determinante (Garganta, 1997; Prudente, 2006; Ribeiro y Silva, 2002; Tavares, 1999).

En consecuencia, este estudio tiene como objetivos analizar, caracterizar y detectar patrones de conductas ofensivos en $2 \mathrm{vs} 2$, relacionando las acciones tácticas con los puestos específicos y su eficacia con los sistemas defensivos donde fueron utilizados, en situación de igualdad numérica en ataque organizado en los partidos de la fase final del Campeonato Europeo Masculino de Balonmano 2012. Con esta investigación se pretende contribuir a mejorar el conocimiento del proceso ofensivo de balonmano masculino al más alto nivel.

\section{Método}

\section{Diseño}

Se analizaron todas las situaciones de juego que se produjeron en ataque organizado con igualdad numérica 6vs6 ocurridas durante los partidos observados del Campeonato Europeo Masculino de Balonmano 2012. Se estudió el comportamiento de dos jugadores de un equipo en una secuencia ofensiva, contra dos defensores. El diseño se encuentra en el cuadrante I, de carácter ideográfico (se observa una unidad), multidimensional (se utiliza un sistema mixto de formato de campo y sistema de categorías), de seguimiento (se observan secuencias en varios partidos a lo largo de una competición) y los datos utilizados son tipo I (sequenciales/multievents) (Anguera, Blanco, Hernández-Mendo y Losada, 2011).

\section{Participantes}

Se observaron 16 partidos, tanto en la fase preliminar como en la final, se han registrado la totalidad de los ataques realizados en igualdad numérica 6vs6 (1028), donde ocurrieron 390 situaciones de juego 2vs2 y un total de 3119 eventos, registrados a partir de los partidos televisados. (Figura 1).

Figura 1. Número de situaciones de juego 2vs2 que ocurren en los partidos observados en el Campeonato de Europa de balonmano masculino de 2012.

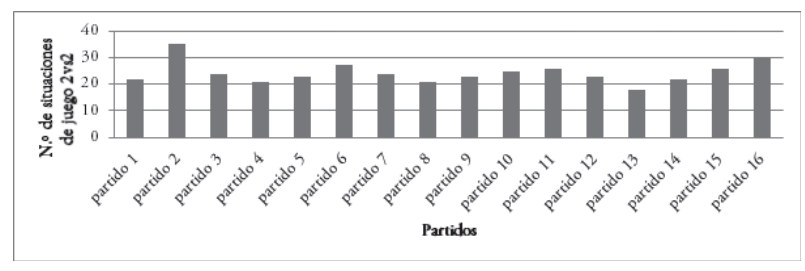

Los criterios de exclusión fueron: 1) los períodos de mayor inobservabilidad superiores al 10\% (Anguera, 1990); 2) los ataques de los equipos que no utilizaron un método de juego de ataque organizado; 3) ataques en los que no había el mismo número de jugadores en ambos equipos y 12 jugadores de campo en el terreno de juego.

\section{Instrumentos}

Con el fin de hacer factible la observación y el análisis de los partidos, se ha diseñado y desarrollado un instrumento de observación basado en estudios previos en el área de análisis del juego de balonmano (Prudente, 2006; Ribeiro, 2002; Silva, 2008; Volossovitch, 2008). A partir del cual se cons- 
truye y valida un instrumento de observación ad hoc para el registro de datos. Es un sistema mixto de formato de campo y sistemas de categorías, que tiene como criterios principales: Organización Defensiva, Tipo de Defensa, Tiempo de Juego,
Marcador, Puestos Específicos, la acción táctica y el Resultado de la Acción (Sousa, Prudente, Sequeira y HernándezMendo, 2014).

Tabla 1. Sistema de observación (Sousa, Prudente, Sequeira y Hernández-Mendo, 2014).

\begin{tabular}{|c|c|c|c|}
\hline Criterios & Categorías & Indicadores & Códigos \\
\hline \multirow[t]{11}{*}{ Organización Defensiva (OD) } & Tipo de Organización & $6: 0 /$ & 6_0 \\
\hline & & $5: 1 /$ & $5 \_1$ \\
\hline & & $4: 2 /$ & $4 \_2$ \\
\hline & & $3: 2: 1 /$ & $3 \_2 \_1$ \\
\hline & & $3: 3 /$ & $3 \_3$ \\
\hline & & $1: 5 /$ & $1 \_5$ \\
\hline & & $5+1$ & $5+1$ \\
\hline & & $4+2 /$ & $4+2$ \\
\hline & & $\mathrm{HxH} /$ & $\mathrm{HxH}$ \\
\hline & Tipo de Defesa & Abierta & Abert \\
\hline & & No Abierta & Nabrt \\
\hline \multirow[t]{6}{*}{ Período de Tiempo de Partido (TJ) } & Período de Tiempo de Partido & $0^{\prime}-10^{\prime}$ minutos de partido & A1 \\
\hline & & $10^{\prime} 01^{\prime \prime}-20^{\prime}$ & $\mathrm{A} 2$ \\
\hline & & $20^{\prime} 01^{\prime \prime}-30^{\prime}$ & A3 \\
\hline & & $30^{\prime} 01^{\prime \prime}-40^{\prime}$ & $\mathrm{B} 1$ \\
\hline & & $40^{\prime} 01^{\prime \prime}-50^{\prime}$ & $\mathrm{B} 2$ \\
\hline & & $50^{\prime} 01-60^{\prime}$ & B3 \\
\hline \multirow[t]{9}{*}{ Resultado de Partido } & Resultado & $\mathrm{E}$ & $E$ \\
\hline & & V1 & $\mathrm{V} 1$ \\
\hline & & $\mathrm{V} 2$ & $\mathrm{~V} 2$ \\
\hline & & V3 & V3 \\
\hline & & V4 & V4 \\
\hline & & D1 & D1 \\
\hline & & $\mathrm{D} 2$ & $\mathrm{D} 2$ \\
\hline & & D3 & D3 \\
\hline & & D4 & D4 \\
\hline \multirow[t]{9}{*}{ Inicio de la acción 2vs2 } & Con el Balón & Con el balón el Pivote en el lado izquierdo & CBPvE \\
\hline & Sin Balón & Con el balón el Pivote en la zona central & CBPvC \\
\hline & Con Parada & Con el balón el Pivote en el lado derecho & CBPvD \\
\hline & & Sin el balón el Pivote en el lado izquierdo & SBPvE \\
\hline & & Sin el balón el Pivote en la zona central & $\mathrm{SBPvC}$ \\
\hline & & Sin el balón el Pivote en el lado derecho & SBPvD \\
\hline & & Con parada el Pivote en el lado izquierdo & CSPvE \\
\hline & & Con stop el Pivote en la zona central & $\mathrm{CSPvC}$ \\
\hline & & Con stop el Pivote en el lado derecho & CSPvD \\
\hline \multirow[t]{5}{*}{ Puestos Específicos } & Puestos Específicos & Lateral izquierdo/Pivote & $\mathrm{LEPv}$ \\
\hline & & Lateral derecho/Pivote & $\mathrm{LDPv}$ \\
\hline & & Central/Pivote & $\mathrm{CPv}$ \\
\hline & & Central/Lateral izquierdo & CLE \\
\hline & & Central/Lateral derecho & CLD \\
\hline \multirow[t]{7}{*}{ Acción Táctica } & Acción Táctica & Bloqueo con Finalización & $\mathrm{Bl}$ \\
\hline & & Bloqueo con Continuidad & $\mathrm{BlC}$ \\
\hline & & Cruce con Finalización & CruzF \\
\hline & & Cruce con Continuidad & CruzC \\
\hline & & Fijar & Fix \\
\hline & & Fijar/Desmarcar & FixDs \\
\hline & & Inversión & Inv \\
\hline
\end{tabular}




\begin{tabular}{lccc}
\hline Criterios & Categorías & Indicadores & Códigos \\
\hline Resultado de la acción & Resultado de la Acción & Gol & $7 \mathrm{G}$ \\
& & Lanzamiento 7 metros & RBl \\
& & Lanzamiento parado en el blocaje & RDGR \\
& & Lanzamiento con la defensa del Portero & RFr \\
& Lanzamiento Fuera & RPsT \\
& Lanzamiento Poste/Travesaño & FT & FA \\
& Falta Técnica & Falta de Atacante & Continuidad de juego \\
\hline
\end{tabular}

\section{Procedimiento}

Los juegos fueron grabados en DVD a partir de las transmisiones televisivas y a continuación pasados a formato AVI con el programa anyvideoconverter y grabados en un disco duro Toshiba STOR.E ALU 2S.

Se han observado todos los juegos, seleccionando todas las secuencias de juego ofensivo en igualdad numérica 6vs6 (para un total de 1028). Se han observado y registrado todas las situaciones de juego 2vs2 (total de 390 acciones) que se han producido.

Detectada la situación de $2 \mathrm{vs} 2$, fue visualizada y registrada desde sus inicio hasta la finalización, la pérdida de balón, o la realización del quinto pase después de la situación táctica desarrollada. Esto constituye al unidad de observación.

El registro de las secuencias ofensivas de las situaciones del juego 2vs2 se realizó utilizando el programa HOISAN, v.1.6 (Hernández-Mendo, López-López, Castellano, Morales-Sánchez y Pastrana, 2012; Hernández-Mendo et al., 2014), que permite realizar todos los cálculos de análisis de calidad del dato y la exportación de los registros a la sintaxis del programa de análisis como SDIS-GSEQ (Bakeman y Quera, 1996, 2011) o SAGT (Hernández-Mendo, Ramos-Pérez y Pastrana, 2012). Además permite realizar análisis secuencial y de coordenadas polares. El tipo de datos utilizado en el registro fue "Multievent».

Ejemplo de una secuencia: 6_0 Nabrt A1 E CBPc CPv Fixar Golo

\section{Muestra}

La muestra se ha compuesto de todas las situaciones de 2vs2 (390) en el que se produjo durante todas las secuencias ofensivas, en igualdad numerica 6vs6 y ataque organizado (1028) pertenecientes a los partidos observados (16).

\section{Calidad de los datos}

El control de la calidad de los datos es un requisito básico en la metodología observacional y permite detectar las fuentes de error o desviación en los datos observados (Blanco y
Anguera, 2003), lo que garantiza la calidad de los datos, su fiabilidad y su sesgo, reduciendo cualquier defecto que pueda comprometer a la investigación (Anguera y Blanco, 2003; Anguera, Blanco, Losada y Hernández-Mendo, 2000; Anguera y Hernández-Mendo, 2013; Blanco y Anguera, 2003; Blanco et al., 2014; Castellano et al., 2000).

Para analizar la calidad de los datos se utilizó la Kappa (K) de Cohen (1960), evaluando la concordancia intra e interobservador, y la teoría de generalización. Los dos observadores que realizan el registro tienen experiencia como entrenadores de balonmano y en calidad de observadores. Antes de comenzar a codificar, los observadores se reunieron y observaron los partidos del Campeonato Mundial Femenino de 2011 y el Campeonato Europeo Masculino de 2012, para llevar a cabo su entrenamiento, discutiendo y consensuando el registro de los diferentes comportamientos con el instrumento de observación construido.

En este estudio, la calidad de los datos se puso a prueba mediante el uso de la prueba de Kappa de Cohen y un análisis de generalizabilidad. La primera se utilizó para estimar la fiabilidad de los observadores y determinar la calidad de los registros obtenidos. Bakeman y Gottman (1989), señalan considerar como valores óptimos de Kappa los superior a 0.70 . El análisis de generalizabilidad se usa complementariamente al anterior (Blanco, Castellano, Hernández-Mendo, Sánchez-López y Usabiaga, 2014; Hernández-Mendo, Montoro-Escaño, Reina-Gómez y Fernández-García , 2012; Perea, 2008; Reina-Gómez, Hernández-Mendo y Fernández-García, 2009) con el fin de excluir diferentes fuentes de variación para los diferentes observadores, instrumento de observación o categorías utilizadas, por lo general constituye un estudio piloto que sirve para preparar un experimento a mayor escala (Blanco, 1989). En un trabajo anterior sobre esta herramienta (Sousa, Prudente, Sequeira y Hernández-Mendo, 2014), se obtuvieron unos resultados excelentes, el índice Kappa de Cohen obtuvo resultados óptimos, entre 0.85 y 1.00, tanto para la fiabilidad intra como para la inter. En cuanto al análisis de generalizabilidad también resulto óptimo, el 97.82\% de la varianza explicada estaba asociada a la faceta categorías (C), y los índices g (relativo y absoluto) demostraron un alto nivel de fiabilidad y de precisión (por encima de 0.9 para el 
modelo $\mathrm{C} / \mathrm{O})$ y buen nivel de ajuste de la categorías en el modelo $\mathrm{O} / \mathrm{C}$.

\section{Análisis estadístico}

Se utiliza estadística descriptiva (frecuencias absolutas y relativas) para caracterizar la muestra y el análisis secuencial de retardos, tanto prospectivo como retrospectivo, base del análisis de coordenadas polares, que permite una representación vectorial de la compleja red de interrelaciones que se establece entre las diferentes categorías constitutivas de un sistema ad hoc (Hernández Mendo, 1999), con p <0.05. Esta técnica analítica permite establecer las diversas relaciones entre una conducta focal y las distintas conductas condicionadas, y construir un mapa vectorial de estas interrelaciones (Prudente, 2006), cuya interpretación depende del cuadrante en el que el vector se encuentre, marcando de este modo la naturaleza de la relación entre las conductas (Anguera, 1997; Castellano y HernándezMendo, 2003; Gorospe, 2000; Hernández-Mendo y Anguera, 1999). En este monográfico hay otros dos artículos que utilizan la técnica de coordenadas polares.

\section{Resultados}

\section{Análisis Descriptiva}

Inicialmente se realizan análisis descriptivos de frecuencias absolutas y relativas. Se indagó sobre cuáles eran las relaciones más frecuentes entre los jugadores de diferentes puestos específicos obteniendo los siguientes resultados: la mayoría de las relaciones observadas fueron «C/Pv» y el «LE/Pv», ambas con un $24 \%$, mientras que la menor relación se observó en «C/LE» con un registro del $13 \%$ de las relaciones entre puestos específicos observados (Figura 2).

Figura 2. Relación entre puestos específicos. Leyenda: C - Central; LE - Lateral izquierdo; LD - Lateral derecho; Pv - Pivote.

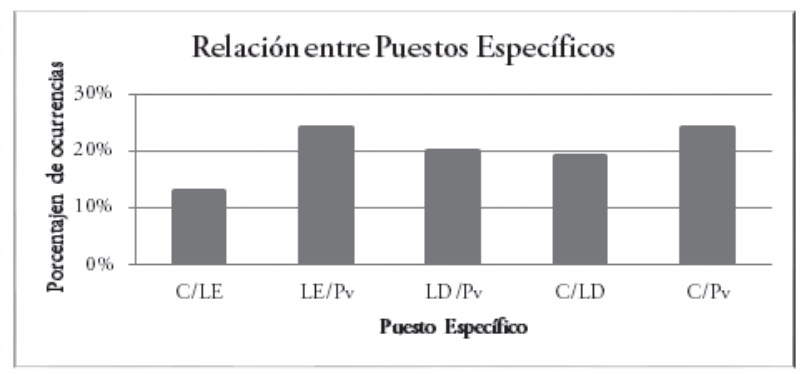

Para comprender el modo de cooperación entre los jugadores registrados de los puestos específicos en las relaciones observadas, se analizan las diferentes acciones tácticas, destacando "Fijar/Desmarcar" (24\%), "Fijar" (23\%) y "Bloqueo" (21\%) como las acciones realizadas con mayor frecuencia, mientras que el "Cruce con Continuidad" (14\%) y el "Cruce con Finalización” (12\%) registraron los valores de ocurrencia más bajos (Figura 3).

Figura 3 - Medios Tácticos Usados en Leyenda: CruzFinal - Cruce con Finalización; CruzCont - Cruce con Continuidad; Fixar - Situación de fijación de los defensores por parte del Pivote, facilitando el lanzamiento de $1^{\text {a }}$ línea; FixDesm - Fijar y Desmarcar (Situación causada por el Pivote, lo que lleva a una salida de uno de los defensores, dejándolo solo para finalizar desde los 6 metros); Bloqueo - Situación de bloqueo por parte del Pivote a un defensor, lo que le permite ganar una posición para finalizar desde los 6 metros.

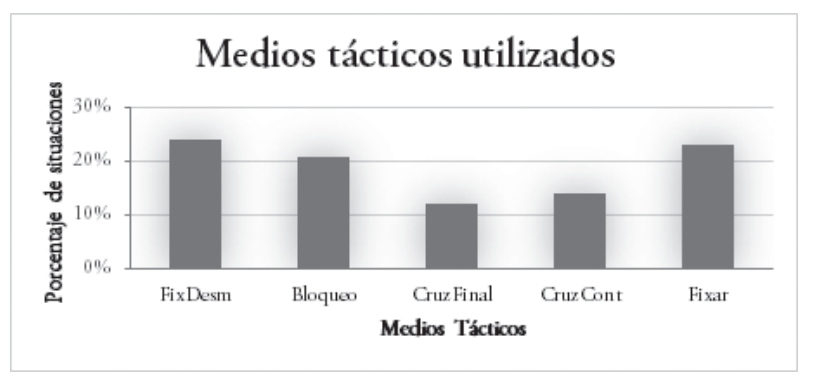

\section{Análisis de coordenadas polares}

La técnica de análisis de coordenadas polares permite situar los vectores en los cuadrantes y determinar sus ángulos, estableciendo así el tipo de relación entre las conductas focales y las conductas condicionadas definidas para cada análisis (Anguera, 1997; Castellano y Hernández-Mendo, 2003; Hernández-Mendo y Anguera, 1999).

Considerando como conductas focales los sistemas defensivos 3:2:1, 5:1 y 6:0, y los puestos específicos de los duelos en $2 \mathrm{vs} 2$ como conductas condicionadas se obtuvieron los siguientes resultados mediante el análisis de coordenadas polares:

a) cuando una conducta focal era el sistema de defensa 3:2:1 (Tabla 2) se activa la conducta condicional de relación $\mathrm{LE} / \mathrm{Pv}(2.36)$ y la $\mathrm{C} / \mathrm{LD}(4.7)$ y se inhibe la conducta $\mathrm{C} / \mathrm{Pv}$ (6.08); 
Tabla 2. Relación entre la conducta focal sistema de defensa 3:2:1, y los puestos específicos como conductas condicionadas.

\begin{tabular}{|c|c|c|c|c|c|c|}
\hline Categoría & Cuadrante & P. Prospectiva & P. Retrospectiva & Ratio & Radio & Ángulo \\
\hline Puestos Específicos_LE/Pv & I & 1.81 & 1.51 & 0.64 & $2.36\left(^{*}\right)$ & 39.98 \\
\hline Puestos Específicos_LD/Pv & III & -0.78 & -0.07 & -0.09 & 0.78 & 185.03 \\
\hline Puestos Específicos_C/LD & I & 3.43 & 3.22 & 0.68 & $4.7\left(^{*}\right)$ & 43.17 \\
\hline Puestos Específicos_C/Pv & III & -4.83 & -3.69 & -0.61 & $6.08\left(^{*}\right)$ & 217.33 \\
\hline Puestos Específicos_C/LE & IV & 1.03 & -0.7 & -0.56 & 1.25 & 325.95 \\
\hline
\end{tabular}

b) cuando la conducta focal fue el sistema defensivo 6:0 (Tabla 3), se activa la conducta $\mathrm{C} / \mathrm{Pv}(4.73)$ y se inhibe las conductas C/LD (3.46) y LE/Pv (2.18). No se obtuvieron re- sultados significativos cuando la conducta focal fue el sistema defensivo 5:1 para las mismas conductas condicionadas.

Tabla 3. Relación entre la conducta focal del sistema de defensa 6:0, y los puestos específicos como conductas condicionadas.

\begin{tabular}{lllllll}
\hline Categoría & Cuadrante & P. Prospectiva & P. Retrospectiva & Ratio & Radio & Ángulo \\
\hline Puestos Específicos_LE/Pv & III & -1.99 & -0.88 & -0.41 & $2.18\left(^{*}\right)$ & 203.98 \\
Puestos Específicos_LD/Pv & IV & 0.36 & -0.01 & -0.03 & 0.36 & 358.10 \\
Puestos Específicos_C/LD & III & -2.36 & -2.53 & -0.73 & $3.46\left(^{*}\right)$ & 226.94 \\
Puestos Específicos_C/Pv & I & 4.05 & 2.44 & 0.52 & $4.73\left(^{*}\right)$ & 31.10 \\
Puestos Específicos_C/LE & II & -0.58 & 0.88 & 0.83 & 1.05 & 123.41 \\
\hline
\end{tabular}

Leyenda: LE/Pv- Relación del Lateral izquierdo con el Pivote; LD/Pv- Relación del Lateral derecho con el Pivote; C/LD- Relación del Central con el Lateral derecho; C/PvRelación del Central con el Pivote; C/LE- Relación del Central con el Lateral izquierdo.

Para el siguiente análisis se considera como conducta focal los cinco medios tácticos más frecuentemente utilizados en los partidos observados («Fijar/Desmarcar»-24\% «Fijar»-23\% «Bloqueo» - 21\% «Cruce con Continuidad» - 14\% y «Cruce con Finalización» - 12\%) para el análisis de coordenadas polares y se considera como las conductas condicionadas los resultados de las acciones, obteniéndose los resultados que se muestran en la Figura 4.
Figura 4. Mapa conductual que expresa las interrelaciones entre la conducta focal "Fijar/Desmarcar" y el resultado de las acciones.

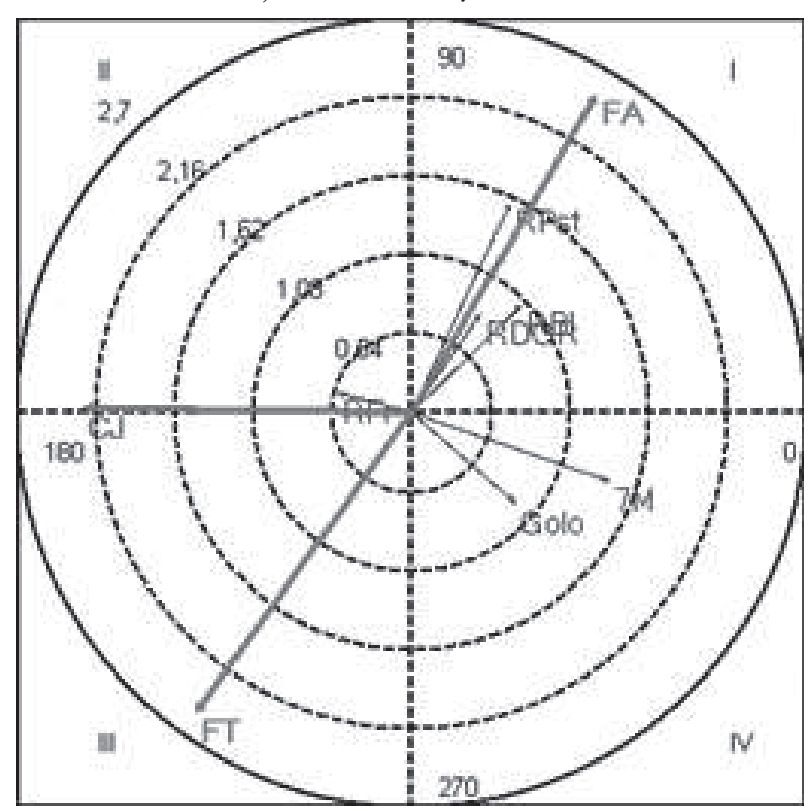

Leyenda: Golo - Gol; $7 \mathrm{M}$ - Lanzamiento 7 metros; RDGR - Lanzamiento con la defensa del Portero; RFr - Lanzamiento fuera; RPsT -Lanzamiento al Poste o el Travesańo; RBl - Lanzamiento parado por Blocaje; FT - Falta Técnica; FA Falta de Atacante; CJ - Continuidad en el Juego. 
De acuerdo con los resultados obtenidos son significativas las relaciones de inhibición con la conducta focal de FT (2.53), y con CJ (2.24) que se activa con la conducta focal. También es significativa la probabilidad de la conducta focal activar la conducta FA (2.50).

La acción táctica de "Fijar» fue igualmente analizada como conducta focal y teniendo como conductas condicionadas las conductas referentes a los resultados. Como se puede observar (Figura 5) sólo se obtuvo un valor significativo: el «Fijar» inhibe a la «Falta de Atacante» (2.37).

Figura 5. Mapa conductual que expresa las interrelaciones entre la conducta focal "Fijar" y el resultado de las acciones.

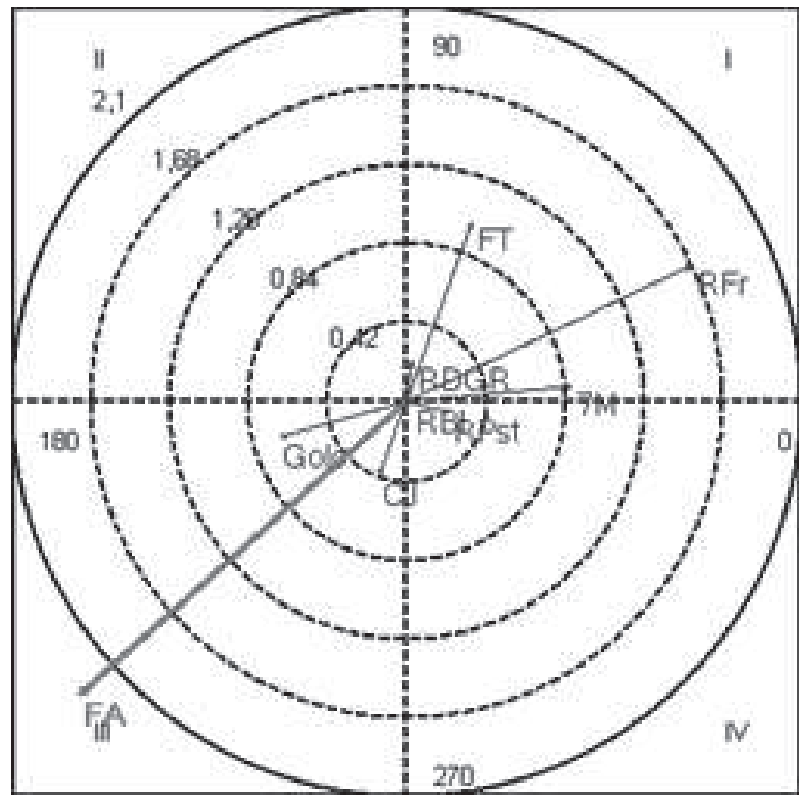

Leyenda: Golo - Gol; 7M - Lanzamiento 7 metros; RDGR - Lanzamiento con la defensa del Portero; RFr - Lanzamiento Fuera; RPsT -Lanzamiento para el Poste o el Travesańo; RBL - Lanzamiento parado por Blocaje; FT - Falta Técnica; FA Falta de Atacante; CJ - Continuidad en el Juego.

El "Bloqueo" fue el tercer medio táctico más observado y se realizó el análisis de coordenadas polares, considerando esta acción como conducta focal, y se detecta una relación de activación de "Gol" (2.22) y "Falta de Atacante" (2.96) y una relación de inhibición de "Lanzamiento 7 metros" (3.15) por este medio táctico, como se muestra en la Figura 6.
Figura 6. Mapa conductual que expresa las interrelaciones entre la conducta focal "Bloqueo" y el resultado de las acciones

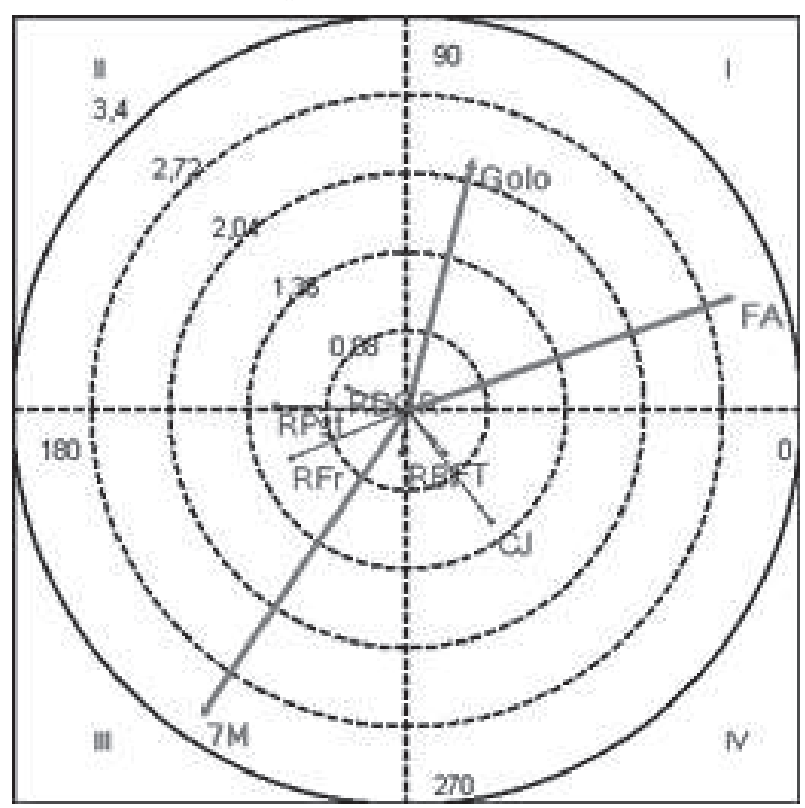

Leyenda: Golo - Gol; 7M - Lanzamiento 7 metros; RDGR - Lanzamiento con la defensa del Portero; RFr - Lanzamiento Fuera; RPsT - Lanzamiento para el Poste o el Travesańo; RBl - Lanzamiento parado por Blocaje; FT - Falta Técnica; FA -

Falta de Atacante; CJ - Continuidad en el Juego.

La acción táctica "Cruce con Continuidad", de acuerdo con los resultados del análisis de coordenadas polares (Figura 7), presenta una relación de inhibición de "Lanzamiento Fuera" (2.35) y de activación de "Lanzamiento al Poste/Travesaño (1.98). Los otros resultados no fueron significativos. 
Figura 7. Mapa conductual que expresa las interrelaciones entre la conducta focal "Cruce con Continuidad" y el resultado de las acciones

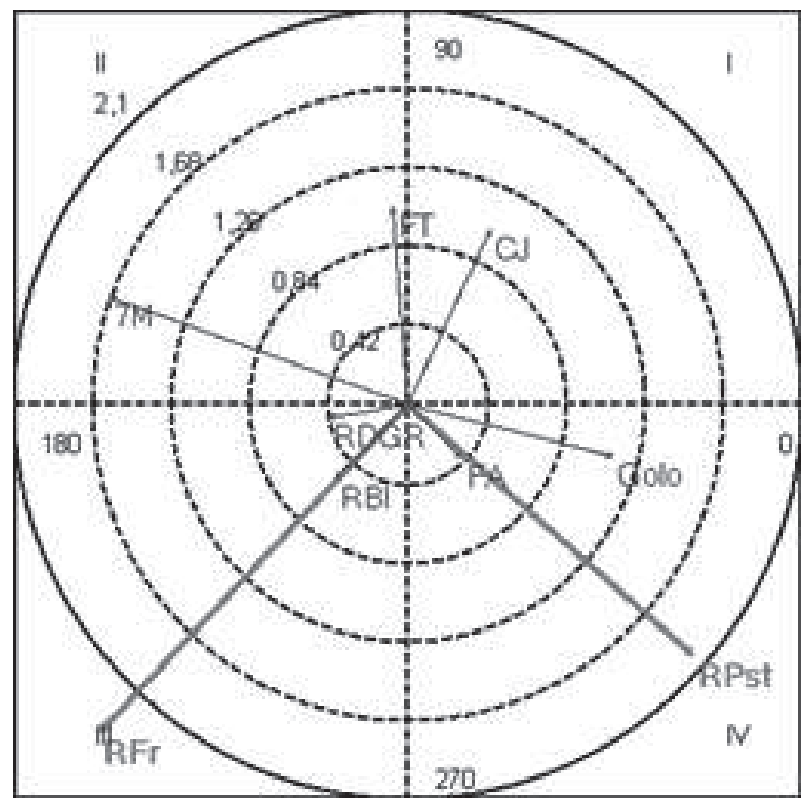

Leyenda: Golo - Gol; 7M - Lanzamiento 7 metros; RDGR - Lanzamiento con la defensa del Portero; RFr - Lanzamiento Fuera; RPsT -Lanzamiento para el Poste o el Travesańo; RBL - Lanzamiento parado por Blocaje; FT - Falta Técnica; FA Falta de Atacante; CJ - Continuidad en el Juego.

Para concluir el análisis de las acciones tácticas como conducta focal y condicionadas las conductas relativas al resultado, se observa los resultados referentes al "Cruce con Finalización” (Figura 8). En la lectura de los resultados obtenidos es posible hablar de la existencia de una relación de activación de "Lanzamiento 7 metros" (1.98), de la "Continuidad del Juego" (2.19) y del "Lanzamiento parado por Portero" (2), siendo que este último es inhibitorio de la conducta focal. La activación de la "Continuidad del Juego" se ha proporcionado por una situación específica, donde fue marcada la falta en el momento del lanzamiento, sin sanción disciplinar. Se registra una relación de inhibición de "Falta de Atacante" (2.84) por esta conducta focal.
Figura 8. Mapa conductual que expresa las interrelaciones entre la conducta focal "Cruce con Finalización" y el resultado de las acciones.

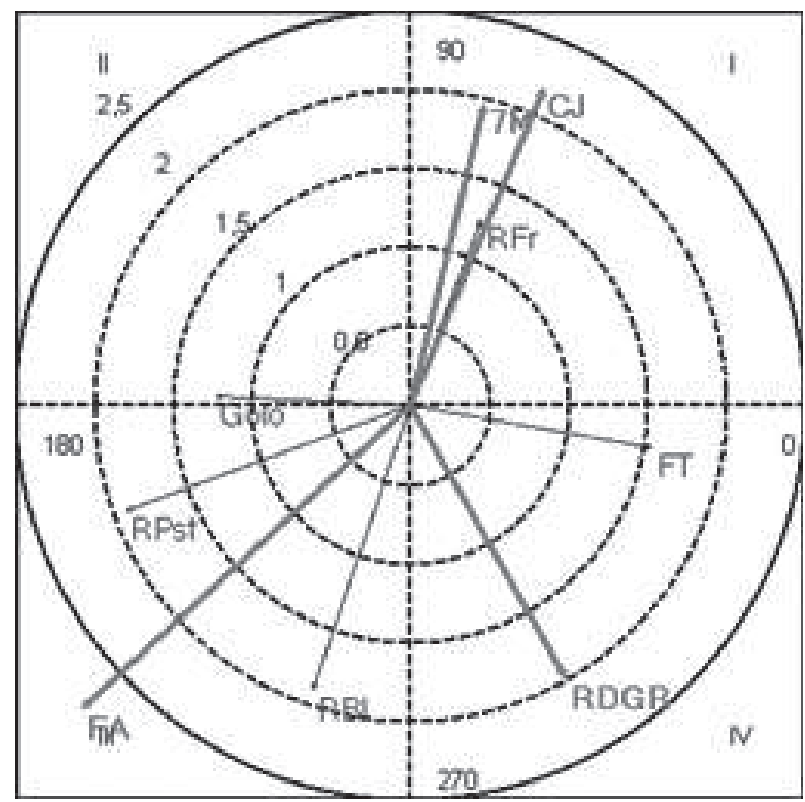

Leyenda: Golo - Gol; 7M - Lanzamiento 7 metros; RDGR - Lanzamiento con la defensa del Portero; RFr - Lanzamiento Fuera; RPsT - Lanzamiento para el Poste o el Travesaño; RBL - Lanzamiento parado por Blocaje; FT - Falta Técnica; FA Falta de Atacante; CJ - Continuidad en el Juego.

Posteriormente, se analiza las relaciones entre los sistemas defensivos y los medios tácticos utilizados en $2 \mathrm{vs} 2$, se comienza por el análisis de las relaciones entre la categoría focal «Sistema 3:2:1» y los diferentes medios tácticos considerados en el estudio. Los resultados muestran que existe una relación significativa de activación entre el «Sistema 3:2:1» y las acciones «Bloqueo con Continuidad» (3.96), «Cruce con Continuidad» (3.25) e «Inversión «(2.87), y la relación inhibitoria significativa entre la conducta focal considerada y la acción de «Fijar» (4.96) (Figura 9). 
Figura 9. Mapa conductual que expresa las interrelaciones entre la conducta focal "Sistema 3:2:1" y las diferentes acciones tácticas.

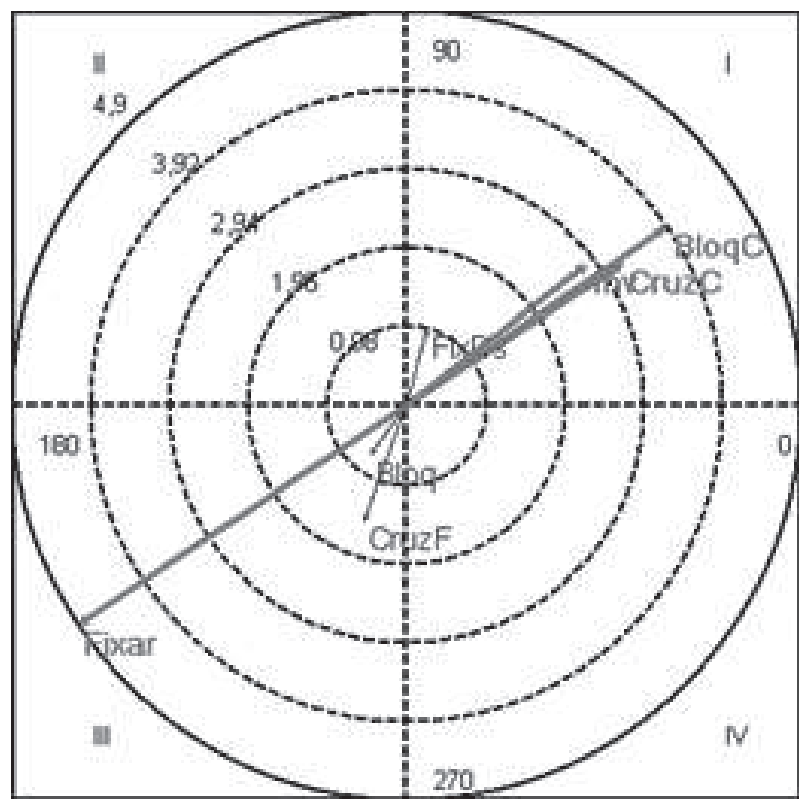

Leyenda: BloqC - Bloqueo con Continuidad; CruzC - Cruce con Continuidad; Inv- Inversión; CruzF - Cruce con Finalización; Bloq - Bloqueo; Fixar-Fijar; FixDs - Fijar/Desmarcar.

Considerando el "Sistema 5:1", como conducta focal se obtienen los resultados que permiten observar que el sistema defensivo tiene una relación inhibitoria significativa con "Bloqueo con Continuidad" (2.66) "Cruce con Finalización" (2.08) y con "Fijar" (2.18) (Figura 10).
Figura 10. Mapa conductual que expresa las interrelaciones entre la conducta focal "Sistema 5:1" y las diferentes acciones tácticas.

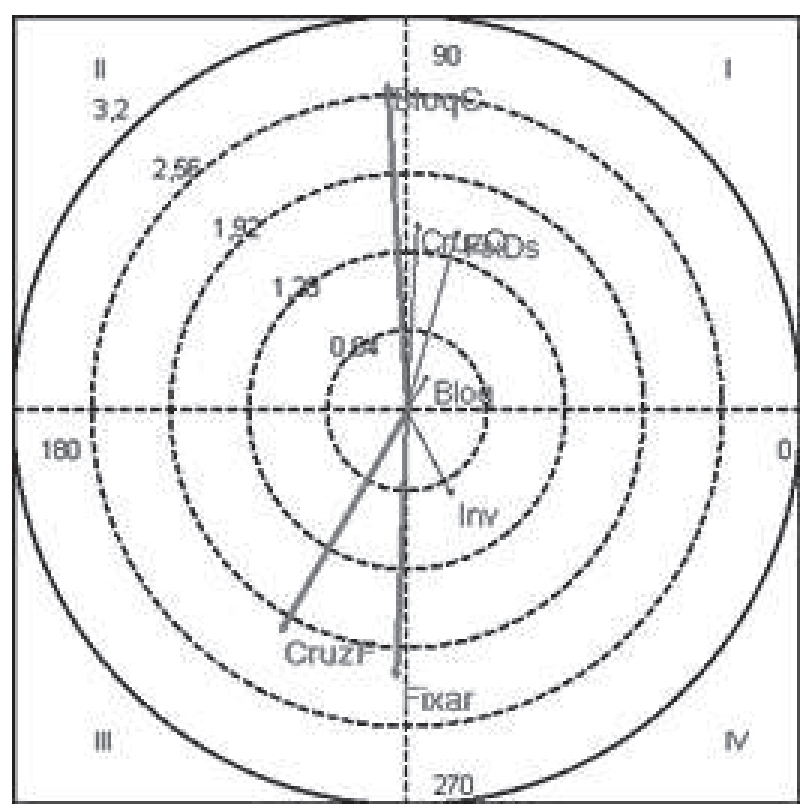

Leyenda: BloqC - Bloqueo con Continuidad; CruzC - Cruce con Continuidad; Inv- Inversión; CruzF - Cruce con Finalización; Bloq - Bloqueo; Fixar-Fijar; FixDs - Fijar/Desmarcar.

Se analiza las relaciones entre el sistema 6:0 y los medios tácticos utilizados, y se obtienen resultados significativos para las relaciones de activación entre el "Sistema 6:0" y las acciones de "Fijar" (4.43) y de "Cruce con Finalización" (3.40), además de una relación significativa inhibitoria entre la conducta focal y las conductas "Cruce con Continuidad" (3.81) y "Bloqueo con Continuidad" (4.08) Figura 11. 
Figura 11. Mapa conductual que expresa las interrelaciones entre la conducta focal "Sistema 6:0" y las diferentes acciones tácticas.

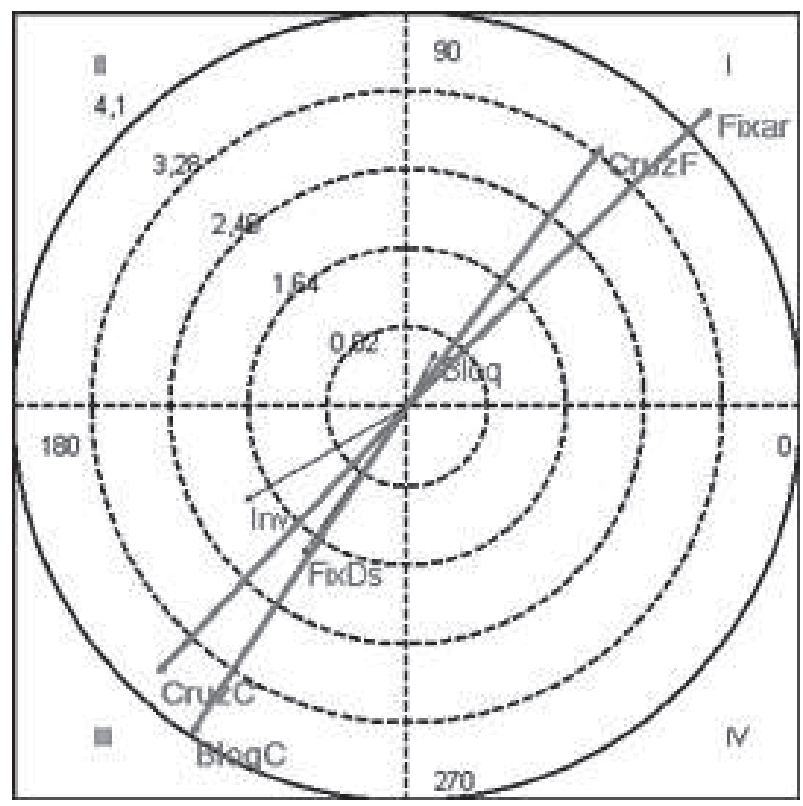

Leyenda: BloqC - Bloqueo con Continuidad; CruzC - Cruce con Continuidad; Inv- Inversión; CruzF - Cruce con Finalización; Bloq - Bloqueo; Fixar-Fijar; FixDs - Fijar/Desmarcar.

\section{Discusión}

En el balonmano existe un predominio de los medios tácticos de equipo (Santos, 2012), por esto, inicialmente se procura verificar cuáles son las relaciones tácticas preferidas entre dos jugadores (Figura 2). Los resultados muestran un predominio de las relaciones entre los jugadores de primera línea (Lateral y Central) y los jugadores de segunda línea, especialmente el Pivote. Estos resultados coinciden con los presentados en el trabajo de Ehret, Johansson, Zovko y Constantini (1995) que señalan que la relación entre el Lateral y el Central con el Pivote es el medio más utilizado por los equipos para sacar el máximo provecho a la calidad del Pivote, incluso seńalando estas relaciones como situaciones de naturaleza táctica muy ricas para superar las defensas en Balonmano. Otro estudio realizado por Santos (1997) en equipos de alto nivel, revela que los elementos que más a menudo llevan a cabo la finalización son el Lateral izquierdo y el Pivote, siendo éste el que presenta el nivel de eficacia más elevado.

Los resultados estimados (Figura 3) confirman que los jugadores que participan en los medios tácticos más utilizados, son el Pivote y los jugadores de la primera línea, donde se encuentra el Lateral Izquierdo. Esta relación entre un jugador de la primera línea con uno de la segunda línea (Pivote) causa dificultades defensivas por las situaciones de incertidumbre, especialmente en las defensas que tienden a salir, teniendo estos intentos el objetivo de vincular el juego exterior con el juego interior (Antón García, 2000). Sin embargo, estos resultados contradicen el estudio de Santos (1997), quien señala que el bloqueo, la pantalla y el intercambio de puesto específico son los medios tácticos de equipo más frecuentes. Todavía en la utilización de bloqueo, Montoya, Moras y Anguera (2013) encuentran una asociación entre los equipos que estaban ganando partidos y la utilización del tiro del pivote. En este estudio, el "Bloqueo» (21\%) es en realidad uno de los medios tácticos más utilizados, pero el «Fijar/Desmarcar» (24\%) y el «Fijar» (23\%) se revelan como los medios tácticos preferidos. Esta situación se explica por la evolución de los sistemas defensivos y el creciente desarrollo de habilidades táctico-técnicas de los jugadores atacantes.

En el análisis de coordenadas polares se comienza analizando la red de relaciones entre los sistemas defensivos y los puestos específicos involucrados en los duelos 2vs2, habiendo logrado resultados significativos cuando se considera como conductas focales los sistemas defensivos 3:2:1 y 6:0 (Figuras $4,5)$ y no se obtiene resultados significativos cuando la conducta focal era el sistema defensivo de 5:1.

En el caso del sistema de 3:2:1 como conducta focal (Figura 4$)$ se puede observar una relación significativa $(>1.96)$ de activación entre la conducta y las relaciones "Central/Lateral derecho" (CLD) y "Lateral izquierdo/Pivote" (LEPv) y una relación de inhibición entre la misma conducta focal y la relación «Central/Pivote» (CPv).

Teniendo en cuenta sólo los resultados significativos (> 1.96) se pueden ver en la Figura 5 que el sistema 6:0 activa la relación "Central/Pivote» $(\mathrm{CPv})$, inhibe las relaciones entre el Lateral izquierdo/Pivote (LEPv) y entre el Central/Lateral derecho (CLD).

Teniendo en cuenta los resultados obtenidos en cuanto al número de ocurrencias de los diferentes medios tácticos se decide optar por el análisis de coordenadas polares considerando como conductas focales los cinco medios tácticos más utilizados en los partidos observados («Fijar/Desmarcar», "Fijar», «Bloqueo», "Cruce con Continuidad" y "Cruce con Finalización») y como conductas condicionadas los resultados de la acción. Los mapas obtenidos se corresponden a las figuras $4,5,6,7$ y 8 .

Lo medio táctico «Fijar/Desmarcar», situación causada por el Pivote, conlleva a una salida de uno de los defensores, dejándolo solo para finalizar desde los 6 metros, se utilizó más como una forma de cooperación en la supuesta situación de juego 2vs2, correspondiente a un medio táctico que implica un jugador de primera línea y el Pivote (figura 4).

Al realizar la acción "Fijar/Desmarcar" es significativa la probabilidad de activar una "Falta de Atacante" e inhibir una "Falta Técnica" y una "Continuidad del Juego" que, a su vez, active el "Fijar/Desmarcar". Este resultado obtenido se explica por el hecho de que el contacto del Pivote con los defensores durante una acción de "Fijar/Desmarcar" puede 
fácilmente terminar en falta (falta de ataque del jugador Pivote). La inhibición de "Falta Técnica" se entiende por el hecho de que se trata de una acción realizada sin balón, en la fase inicial cuando se le da el pase al Pivote éste se encuentra desmarcado, lo que facilita la recepción. Dado que se trata de una acción que tiene como objetivo la creación de facilitar el lanzamiento desde primera línea, u ocasionar la salida de uno de los defensores, se crea una situación de finalización de 6 metros por el Pivote, que no favorece la continuidad del ataque. Una acción táctica "Fijar/Desmarcar" facilita la posibilidad del lanzamiento desde 6 metros, por lo que es muy eficaz cuando se produce. Sin embargo, los valores obtenidos relativos al lanzamiento (Gol, RDGR, RFr) no son significativos, al contrario de lo que cabría esperar.

La acción táctica de "Fijar" es también una acción que implica al jugador Pivote y a un jugador de primera línea. Se trata de una acción en la que ocurre una situación de fijación de los defensas por parte del Pivote, facilitándose el lanzamiento desde primera línea. Sin embargo, el mapa obtenido permite dar cuenta de que, de una manera significativa, el «Fijar» sólo inhibe a «Falta de Atacante» porque a diferencia del «Bloqueo» y de "Fijar/Desmarcar" la lucha con el defensor para liberarse de marcaje o bloqueo no existe. Por el contrario a lo que cabría esperar valores relativos a la finalización, como el lanzamiento, no fueron significativos.

El "Bloqueo" permite crear situaciones que facilitan la finalización y la liberación del jugador marcado. Estos resultados están de acuerdo con los estudios que apuntan a la importancia de los conceptos de trabajo en equipo en los partidos de Balonmano (Sequeira, 2012) entre dos jugadores y recurrir al uso del Pivote (Santos, 2012) que causan desequilibrios determinantes para la obtención del éxito en el equipo. Sin embargo, se trata de una acción táctica que exige una correcta acción de bloquear sin infringir las reglas, lo que no ocurre, provoca un elevado nivel de faltas atacantes. El bloqueo, se realiza en un espacio muy pequeńo, con los jugadores en movimiento, tratando de ganar la posición, provoca enfrentamientos entre ellos, a menudo conduce al bloqueador a infringir las reglas del juego. Asimismo, el mapa que se obtiene cumple con las expectativas, que muestran una relación de activación de "Bloqueo" con el "Gol" y con la "Falta de Atacante" y una relación inhibitoria con "Lanzamiento 7 metros".

La acción táctica "Cruce con Continuidad" presenta una relación de inhibición con "Lanzamiento Fuera" y de activación con "Lanzamiento al Poste/Travesaño", no siendo significativos los restantes resultados.

En cuanto a la acción táctica "Cruce con Finalización" (Figura 8) se detecta una relación de activación de «Lanzamiento 7 metros», y "Continuidad del Juego» y de «Lanzamiento con la defensa del Portero" de este último inhibitoria con «Cruce con Finalización». Como se puede observar en el mapa existe una relación inhibitoria de "Falta de Atacante" con "Cruce con Finalización". Los resultados confirman que existe una probabilidad significativa de "Cruce con Finalización", que se asocia a una situación de lanzamiento fallido por parada del portero, que refuerza los estudios anteriores que relacionan este medio táctico con la ineficacia del ataque (Antón García, 1994; Prudente, 2006) o asociado a los equipos perdedores (Silva, 2008).

Se puede explicar esta situación por la especialización de los jugadores defensores, así como mediante el aumento de la asistencia mutua y la cooperación entre los defensores y el portero. Dado que el cruce está asociado con una finalización desde primera línea, se puede decir que los resultados confirman el estudio Prudente (2006), que establece que cuando existe una colaboración entre el portero y la defensa, la probabilidad de que se produzca una parada de portero es significativa.

La relación de activación de "Lanzamiento 7 metros" resulta de la posibilidad de cruce que permite al jugador que se beneficie de ella, una situación de fijación de dos adversarios, obteniendo un lanzamiento con la penetración del compañero al que le pasa el balón. Cuando este jugador es objeto de falta mientras realiza la penetración, significa obtener lanzamiento de 7 metros, ya que se trata de un jugador, con una posición ganadora en relación con el defensor, sobre el cual se ha cometido una falta intentando impedir el lanzamiento.

Tras el análisis de las relaciones entre los resultados de la utilización de las diferentes acciones y considerando éstas como categorías focales, es interesante comprobar la red de relaciones entre los sistemas defensivos y los medios tácticos utilizados en 2vs2, se empieza por el análisis de las relaciones entre la categoría focal «Sistema 3:2:1» y los diferentes medios tácticos (Figura 9).

El mapa que se obtiene muestra la existencia de una relación significativa de activación entre el «Sistema 3:2:1» y las acciones «Bloqueo con Continuidad», "Cruce con Continuidad»e «Inversión», así como una relación significativa inhibitoria entre la conducta focal considerada y la acción de «Fijar». Cuando se considera el «Sistema 5:1» como conducta focal, se obtienen los resultados que figuran en el mapa conductual (Figura 10) y se verifica que este sistema defensivo tiene una relación inhibitoria significativa con el «Bloqueo con Continuidad», "Cruce con Finalización» y con «Fijar».

No se obtienen resultados significativos relativos a la red de relaciones con esta conducta focal con los medios tácticos utilizados durante las situaciones de juego $2 \mathrm{vs} 2$.

Por último se analiza la red de relaciones entre el sistema 6:0 y los medios tácticos utilizados, y se obtiene el siguiente mapa conductual (Figura 11) donde se puede observar la relación de activación entre el "Sistema 6:0" y las acciones "Fijar" y de "Cruce con Finalización", un resultado contrario si se compara con lo obtenido en " $3: 2: 1$ ” y en " $5: 1$ ”, donde la 
conductas focales inhiben estas acciones tácticas. Igualmente, se puede observar una relación significativa inhibitoria entre la conducta focal y las conductas "Cruce con Continuidad" y "Bloqueo con Continuidad".

Los resultados parecen querer indicar que, delante una defensa sin profundidad, los jugadores en $2 \mathrm{vs} 2$, optan por una cooperación que permita la finalización con la primera línea, mientras que en el " $5: 1$ ” y en el " $3: 2: 1$ ” los jugadores optan por las acciones que permitan la penetración con finalización por el Pivote, aunque, en el caso de 5:1, los resultados no son significativos, es, sin embargo, clara preferencia de cara a la defensa 3:2:1.

\section{Conclusiones}

Este estudio tenía como objetivos analizar, caracterizar y detectar patrones de conductas ofensivos en $2 \mathrm{vs} 2$, relacionando las acciones tácticas y su eficacia con los sistemas defensivos en los que fueron utilizados, basándose en el análisis de coordenadas polares.

Se observa que en las principales relaciones entre dos jugadores (Lateral izquierdo/Pivote, Central/Pivote y Lateral derecho/Pivote), el Pivote es uno de los dos jugadores que intervienen, situación que corrobora Santos (2012) que afirma que la actividad del Pivote puede influir en una perspectiva táctico-técnica y directa o indirectamente en los resultados de las acciones ofensivas.

Asimismo, se observa que los diferentes sistemas defensivos activan las relaciones de cooperación entre los diferentes puestos específicos, así como, fueron diferentes las acciones tácticas activadas en contra de los diferentes sistemas defensivos.

En varios patrones se detecta, que se recurre a la utilización del Pivote, en particular, a través de "Fijar", "Fijar/Desmarcar" y de "Bloqueo", con diferentes resultados. El "Bloqueo" fue la única acción que activó "Gol”, si bien se puede considerar que la acción "Cruce con Finalización”, por activar, de forma significativa "Lanzamiento 7 metros", fue igualmente una acción donde existía eficacia.

Estos resultados pueden fomentar a una reflexión de los entrenadores sobre la planificación del entrenamiento, teniendo en cuenta las nuevas tendencias, con el fin de seleccionar las acciones más adecuadas a las situaciones del balonmano actual, tanto dirigido a las tareas de equipo, los diferentes contextos del juego en ataque.

\section{Aplicaciones prácticas}

Esta técnica de análisis ha permitido comparar las relaciones que se establecen en la utilización de los medios tácticos grupales considerando los puestos específicos y los sistemas defensivos. El establecimiento de un mapa conceptual de las relaciones establecidas entre la categoría considerada como focal y todas las demás categorías condicionadas permite dar cuenta de una manera clara de la evolución de dichas relaciones tanto en su significación (longitud del vector) como en la naturaleza de las mismas (cuadrante en el que se ajustan), lo que, sin duda, puede tener implicaciones táctico-técnicas para los entrenadores. Asimismo, se sugiere el entrenamiento de los medios tácticos grupales, preferencialmente los que promueven la integración del Pivote, como el «Fijar», «Fijar/Desmarcar» y el «Blocaje». Igualmente, se sugiere el entrenamiento de lo «Blocaje» para reducir la posibilidad de «Falta de Atacante».

Los resultados de la presente investigación han sido parcialmente financiados por los proyectos: Observación de la interacción en deporte y actividad física: Avances técnicos y metodológicos en registros automatizados cualitativos-cuantitativos, subvencionado por la Secretaría de Estado de Investigación, Desarrollo e Innovación del Ministerio de Economía y Competitividad [DEP2012-32124], durante el trienio 2012-2015; y "Evaluación Psicosocial en Contextos Naturales: Deporte y Consumo (SEJ 444)", financiado por la Junta de Andalucía (Consejería de Innovación, Ciencia y Empresa).

\section{Referencias}

1. Anguera, M.T. (1990). Metodología observacional. En J. Arnau, M. T. Anguera y J. G. Benito (Eds.), Metodología de la investigación en ciencias del comportamiento (pp. 125-236). Murcia: Universidad de Murcia.

2. Anguera, M.T. (1997). From prospective patterns in behavior to joint analysis with a retrospective perspective. Colloque sur invitation "Méthodologie d'analyse des interactions sociales». Paris: Université de la Sorbonne.

3. Anguera, M.T., y Blanco, A. (2003). Registro y Codificación del comportamiento deportivo. En A. Hernández-Mendo (Coord.), Psicología del Deporte. (Vol. 2), Metodología (pp. 6-34). Buenos Aires: Efdeportes (www.efdeportes.com).

4. Anguera, M.T., y Hernández-Mendo, A. (2013). La metodología observacional en el ámbito del deporte. E-balonmano.com: Revista de Ciencias del Deporte 9(3), 135-160. http://www.e-balonmano.com/ojs/ index.php/revista/index
5. Anguera, M.T., Blanco, A., Losada, J.L. y Hernández-Mendo, A. (2000). La metodología observacional en el deporte: conceptos básicos. Lecturas: EF y Deportes. Revista Digital, 24.

6. Anguera, M.T., Blanco, A., Hernández-Mendo, A. y Losada, J. (2011) Diseńos observacionales: ajuste y aplicación en psicología del deporte. Cuadernos de Psicología del Deporte, 11(2), 63-76.

7. Antón García, J. (1994). Balonmano: Metodología y alto rendimiento (1a ed.). Barcelona: Paidotribo.

8. Antón García, J. (2000). Balonmano. Perfeccionamiento e Investigación. Zaragoza. INDE publicaciones.

9. Bakeman, R., y Gottman, J.M. (1989). Observación de la interacción: Introducción al análisis secuencial. Madrid: Ediciones Morata, S.A.

10. Bakeman, R. y Quera, V. (1996). Análisis de la interacción. Análisis secuencial con SDIS y GSEQ. Madrid: RA-MA. 
11. Blanco, A. (1989). Fiabilidad y generalización de la observación conductual. Anuario de Psicología, 43(4), 5-32.

12. Blanco, A., y Anguera, M.T. (2003). Calidad de los datos registrados en el ámbito deportivo. En A. Hernández-Mendo (Coord.), Psicologia del Deporte (Vol. 2). Metodologia (p.35-73). Buenos Aires: Efdeportes (www.efdeportes.com).

13. Blanco, A., Castellano, J., Hernández-Mendo, A., Sánchez-López, C. R. y Usabiaga, O. (2014). Aplicación de la TG en el deporte para el estudio de la fiabilidad, validez y estimación de la muestra. Revista de Psicología del Deporte, 23(1), 131-137.

14. Castellano, J. y Hernández-Mendo, A. (2003). El análisis de coordenadas polares para la estimación de relaciones en la interacción motriz en fútbol. Psicothema, 15(4),569-574.

15. Castellano, J., Hernández Mendo, A., Gómez de Segura, P., Fontetxa, E. y Bueno, I. (2000). Sistema de codificación y análisis de la calidad del dato en el fútbol de rendimiento. Psicothema, 12(4), 636-641.

16. Cohen, J. (1960). A coefficient of agreement for nominal scales. Educational and Psychological Measurement, 20, 37-46.

17. Gorospe, G. (2000). Observación y Análisis de la acción de juego en el tenis de individuales: Aportaciones del análisis secuencial y de las coordenadas polares. Revista Digital Efdeportes, 5(21),1-2. Disponible en: http://www.efdeportes.com/efd21a/tenis.htm

18. Ehret, A., Johansson, B., Zovko, Z. y Constantini, D. (1995). Dossier special mondial. L'analyse du jeu par les meilleurs entraineurs. Approches Du Handball, 27, 7-16.

19. Garganta, J. (1997). Modelação Táctica no jogo de Futebol. Porto: J. Garganta. Dissertação de Doutoramento (Não publicada). FCDEFUP. Porto.

20. Hernández-Mendo, A. (1999). Observación y Deporte. In M. T. Anguera (Ed.), Observación en Deporte y conducta cinésico-motriz: Aplicaciones. Barcelona: Edicions Universitat de Barcelona.

21. Hernández-Mendo, A. y Anguera, M.T. (1999). Aportaciones de análisis de coordenadas polares a los deportes de equipo. En F. Guillén (Ed.), La Psicología del Deporte en España al final del milenio (pp. 169-175). Las Palmas: Universidad de Las Palmas de Gran Canaria.

22. Hernández-Mendo, A., Castellano, J., Camerino, O., Jonsson, G., Blanco-Villaseñor, A., Lopes, A, y Anguera, M. T. (2014). Programas informáticos de registro, control de calidad del dato, y análisis de datos. Revista de Psicología del Deporte, 23(1), 111-121.

23. Hernández-Mendo, A., Montoro-Escaño, J., Reina-Gómez, A. y Fernández-García, J.C. (2012). Desarrollo y optimización de una herramienta observacional para el bloqueo en voleibol. Revista Iberoamericana de Psicología del Ejercicio y el Deporte, 7(1), 15-32.

24. Hernández-Mendo, A., Ramos-Pérez, F., y Pastrana, J.L. (2012). SAGT: Programa informático para análisis de Teoría de la Generalizabilidad. SAFE CREATIVE Código: 1204191501059.

25. Hernández-Mendo, A., López-López, J.A., Castellano, J., MoralesSánchez, V. y Pastrana, J.L. (2012). Hoisán 1.2: Programa informático para uso en Metodología observacional. Cuadernos de Psicología del Deporte, 12 (1), 55-78.

26. Montoya, M., Moras, G. y Anguera, M.T. (2013). Análisis de las finalizaciones de los extremos en balonmano. Apunts. Educación Física y Deportes, 113(3), 52-59.
27. Perea, A. (2008). Análisis de las acciones colectivas en el fútbol de rendimiento. Tesis Doctoral sin publicar, Universidad del País Vasco, San Sebastián, España.

28. Prudente, J. (2006). Análise da performance táctico-técnica no Andebol de alto nível: estudo das açóes ofensivas com recurso à análise sequencial. Tese de Doutoramento (Não publicada). Universidade da Madeira. Funchal.

29. Reina-Gómez, A., Hernández-Mendo, A. y Fernández-García, J.C. (2009). Multi-facet design for goal scoring in SOCCER-7. Quality y Quantity,44(5), 1025-1035. DOI 10.1007/s11135-009-9253-8

30. Ribeiro, B. y Silva, J. (2002). A importância dos meios tácticos de grupo ofensivos na obtenção do golo em andebol. Um estudo com recurso à análise sequencial. Comunicação apresentada no II Congresso Nacional de técnicos Especialistas en Balonmano: "La estructuración del juego de ataque", Cáceres.

31. Román Seco, J. (2005). Los inicios del siglo XXI: Evolución y tendencias del juego. Comunicação apresentada em Seminario Asociación de Entrenadores de Balonmano. Zaragoza.

32. Santos, L. (1997). Processo de selecção em andebol: um estudo exploratório com treinadores brasileiros. Dissertação de mestrado. FCDEF - UP Porto.

33. Santos, L. (2004). Tendências evolutivas do jogo de andebol: estudo centrado na análise da performance táctica de equipas finalistas em Campeonatos do Mundo e Jogos Olimpicos. Tese de doutoramento. (não publicada) FCDEF -UP. Porto.

34. Santos, M. (2012). O jogador pivot no jogo de Andebol: Análise da sua actividade no processo ofensivo das Selecçōes Nacionais masculinas no Campeonato Mundial 2007, Campeonato Europeu e Jogos Olimpicos 2008. Tese de Doutoramento (não publicada). Facultade de Ciencias do Deporte e a Educación Física. Universidade da Coruña. Coruña.

35. Sequeira, A. (2012). Análisis de los Factores de Eficacia de las Acciones de Pré-fnalización y Finalización en Ataque Organizado en el Balonmano de Alto nivel. Tesis Doctoral. FCD - Universidad de Castilla-La Mancha. Toledo. España.

36. Sevim, Y. y Bilge, M. (2005). The comparison of the last Olympic, World anEuropean men Handball Championships and the current developments in World Handball. Comunicação apresentada em 2005 Youth Coaches' Course.

37. Silva, J. (2008). Modelação Táctica do Processo Ofensivo em AndebolEstudo de situações de igualdade numérica, 7 vs 7 , com recurso à Análise Sequencial. Tese de Doutoramento (Não publicada). FCDEF - UP. Porto.

38. Sousa, D., Prudente, J., Sequeira, P. y Hernández-Mendo, A. (2014). Análise da qualidade dos dados de um instrumento para observaçáo do 2 vs 2 no andebol. Revista Iberoamericana de Psicología del Ejercicio y el Deporte, 9 (1), 173-190.

39. Tavares, F. (1999). A investigação da componente táctica nos Jogos Desportivos Colectivos: conceitos e ilustraçōes. In F. Tavares (Ed.), Estudo dos Jogos Desportivos. Concepçóes, metodologias e instrumentos (pp. 7-13). Porto: C.E.J.D.- FCDEF

40. Volossovitch, A. (2008). Análise dinâmica do jogo de andebol. Estudo dos factores que influenciam a probabilidade de marcar golo. Tese de Doutoramento (Não publicada). FMH-UTL. Lisboa. 